\title{
Article \\ HPV Genotyping by Molecular Mapping of Tissue Samples in Vaginal Intraepithelial Neoplasia (VaIN) and Vaginal Squamous Cell Carcinoma (VaSCC)
}

\author{
Shitai Zhang ${ }^{1,2}$, Mayumi Saito ${ }^{1}$, Kaori Okayama ${ }^{3}$, Mitsuaki Okodo ${ }^{4}{ }^{\infty}$, Nozomu Kurose ${ }^{5}$, Jinichi Sakamoto ${ }^{1}$ \\ and Toshiyuki Sasagawa ${ }^{1, *}$ \\ 1 Department of Obstetrics and Gynecology, Kanazawa Medical University, Kahoku-gun 920-0293, Japan; \\ zhangshitai@126.com (S.Z.); m-saito@kanazawa-med.ac.jp (M.S.); sakamoto@kanazawa-med.ac.jp (J.S.) \\ 2 Department of Obstetrics and Gynecology, Shengjing Hospital of China Medical University, \\ Shenyang 110004, China \\ 3 School of Medical Technology, Faculty of Health Science, Gumma Paz University, Takasaki 320-0006, Japan; \\ okayama@paz.ac.jp \\ 4 Department of Medical Technology, Faculty of Health Sciences, Kyorin University, Mitaka 181-8611, Japan; \\ ohkoudom@ks.kyorin-u.ac.jp \\ 5 Department of Pathology and Laboratory Medicine, Kanazawa Medical University Hospital, \\ Kahoku-gun 920-0293, Japan; k-nozomu@kanazawa-med.ac.jp \\ * Correspondence: tsasa@kanazawa-med.ac.jp; Tel.: +81-76-218-8143; Fax: +81-76-286-2629
}

Citation: Zhang, S.; Saito, M.; Okayama, K.; Okodo, M.; Kurose, N.; Sakamoto, J.; Sasagawa, T. HPV Genotyping by Molecular Mapping of Tissue Samples in Vaginal Intraepithelial Neoplasia (VaIN) and Vaginal Squamous Cell Carcinoma (VaSCC). Cancers 2021, 13, 3260. https://doi.org/10.3390/ cancers 13133260

Academic Editors: Brian Gabrielli and Kevin Gaston

Received: 21 April 2021

Accepted: 25 June 2021

Published: 29 June 2021

Publisher's Note: MDPI stays neutral with regard to jurisdictional claims in published maps and institutional affiliations.

Copyright: (c) 2021 by the authors. Licensee MDPI, Basel, Switzerland. This article is an open access article distributed under the terms and conditions of the Creative Commons Attribution (CC BY) license (https:// creativecommons.org/licenses/by/ $4.0 /)$.
Simple Summary: HPV genotypes were determined in 63 vaginal intraepithelial neoplasia (VaIN) and 7 vaginal squamous cell carcinomas (VaSCC). Of these, 37 cases had VaIN alone, and 26 cases had both VaIN and cervical intraepithelial neoplasia (CIN) or condyloma. HPV typing was performed in scraped cells by Genosearch-31 and in the archived tissues by uniplex E6/E7 PCR. In a total of 49 VaIN1, 17 VaIN2/3, and 7 VaSCC tissues, the prevalence of HPV was $91.2 \%$ in VaIN and $85.7 \%$ in VaSCC. Comparing HPV results in scraped cell and tissue, $46.2 \%$ of high-risk (HR) types and $68.1 \%$ of any HPV types that had been identified in cell samples were not present in corresponding tissues. HPV types in VaIN and CIN lesions differed in $92.3 \%$ of cases with multiple lesions. These results suggest that there are many preclinical HPV infections in the vagina or the cervix, and VaIN and CIN are independently developed. The manual microdissection procedure of tissue revealed one HPV type in one lesion. The vagina appears to be the reservoir for any mucosal HPV type, and HR- or pHR-HPV types are causative agents for vaginal malignancies.

Abstract: HPV genotypes were determined in 63 vaginal intraepithelial neoplasia (VaIN) and 7 vaginal squamous cell carcinomas (VaSCC). Of these, 37 cases had VaIN alone, and 26 cases had both VaIN and cervical intraepithelial neoplasia (CIN) or condyloma. HPV typing was performed in scraped cells by Genosearch-31 (GS-31) and in the archived tissues by uniplex E6/E7 PCR. In a total of 49 VaIN1, 17 VaIN2/3, and 7 VaSCC tissues, the prevalence of HPV was $91.2 \%$ in VaIN (VaIN1: 87.8\%, VaIN2/3: 100\%) and 85.7\% in VaSCC. Comparing HPV results in scraped cell and tissue, $46.2 \%$ of high-risk (HR) types and $68.1 \%$ of any HPV types that had been identified in cell samples were not present in corresponding tissues. HPV types in VaIN and CIN lesions differed in $92.3 \%(24 / 26)$ of cases with multiple lesions. These results suggest that there are many preclinical HPV infections in the vagina or the cervix, and VaIN and CIN are independently developed. The manual microdissection procedure of tissue revealed one HPV type in one lesion. Seventeen HPV types, including high-risk (HR), possible high-risk ( $\mathrm{pHR}$ ), and low-risk (LR), were identified in 43 VaIN1 lesions. In higher grade lesions, six HR (HPV16, 18, 51, 52, 56, 58), one pHR (HPV66), and one LR (HPV42) HPV types were identified in 17 VaIN2/3, and six HPV types, including HPV16, 45, 58, and 68 (HR), and HPV53 and 67 (pHR), were detected in each case of VaSCC. The vagina appears to be the reservoir for any mucosal HPV type, and HR- or PHR-HPV types are causative agents for vaginal malignancies. 
Keywords: human papillomavirus; vaginal intraepithelial lesion; uniplex E6/E7 PCR; manual microdissection

\section{Introduction}

Human papillomavirus (HPV) has been proven to be the causative agent of cervical cancer and its precancerous lesions, this virus belongs to the Papillomaviridae family, which is a small, non-enveloped type, double-strand DNA (dsDNA) virus group [1]. To date, more than 200 types had been identified on the basis of DNA sequence data [2,3], and about $50 \mathrm{HPV}$ types have been identified in the mucosal epithelium of the uterine cervix, the vagina, and the vulva [4]. A group of approximately one dozen forms of HPV comprise the main etiologic factors for the development of neoplasms of the lower genital tract of women. It is estimated that $70-80 \%$ of all women are infected with cervical $\mathrm{HPV}$ at some point during their lifetime, and $90 \%$ of these infections can be eliminated within a few years $[5,6]$. However, $10 \%$ of women fail to clear the virus, and long-term persistent infection induces cervical cancer [6,7]. On the basis of their association with premalignant and malignant lesions, $13 \mathrm{HPV}$ types (HPV16, 18, 31, 33, 35, 39, 45, 51, 52, $56,58,59$, and 68) have been defined as high-risk (HR), although HPV68 is categorized as a probable high-risk type [8]. An additional nine HPV types (HPV26, 30, 34, 53, 66, $67,70,73$, and 82), which belong to alpha-5, 6, 7, 9 and 11 genera, are thought to be possible high-risk HPV (pHR) types because these types are identified in cancer tissue [9]. HR-HPV encodes two oncoproteins, E6 and E7, which are continuously transcribed in premalignant and malignant tissues. The E7 oncoprotein binds to the tumor-suppressive retinoblastoma protein $(\mathrm{pRb})$, and this interaction facilitates host cell proliferation by inhibition of the function of $\mathrm{pRb}$. The E6 oncoprotein also down-regulates p53, which functions as a gatekeeper protein that regulates cell proliferation and induces apoptosis of the cells damaged by UV-stimualtion [10]. The E6 oncoprotein also inhibits a group of proteins having the PDZ-motiff [11,12]. Thus, E6 and E7 act synergistically to drive host cell proliferation without regulation, and transform these cells into cancer cells. However, it is thought that such actions are generally not present in low-risk (LR) HPV types.

Although the causal relationship between HPV infection and cervical SIL and cancer development has been confirmed by many studies, the role of HPV in the vagina is not well understood. One reason for this is that vaginal squamous cell carcinoma is a rare disease, and HPV-positivity in the cancer $(60-70 \%)$ is less than that in cervical squamous cell cancer (more than 95\%) [13,14]. Another report showed that 93.6\% of vaginal intraepithelial lesions (VaINs) are positive for HPV [15]. We have also reported that various HPV types are identified in the vaginal lesions, and HPV is positive in $84 \%$ of VaIN1 cases and $100 \%$ of VaIN2/3 cases [16]. Highly sensitive HPV testing has shown that most VaIN cases are positive for HPV, although a few cases of VaIN1 have been shown to be negative for HPV $[13,16]$. During the carcinogenic process after HPV infection, in addition to the HPV genotype, the infection site may also be important. Many studies suggest that infection in certain cell populations located in the squamous-columnar junction (SCJ) of the cervix is a crucial step for cancer development [17]. We have previously reported that multiple HPV types can be frequently identified in the cervical cell samples. ${ }^{16}$ However, it has been reported that one HPV type is found in one lesion, including cervical cancer and high-grade SILs (CIN2 or CIN3) [18]. This suggests that scraped cell samples from the cervix are likely to contain different cell populations from various lesions, including the vaginal lesions. Therefore, cell samples are not suitable for the determination of the HPV type responsible for the target lesion. Recently, it was found that a combination of laser capture microdissection (LCM) and highly sensitive HPV testing may be the most effective test to clarify the relationship between a certain HPV type and the related lesion [19]. Alternatively, Snow AN et al. [20] also introduced manual microdissection with small FFPE (formalin fixed and paraffin embedded) tissue specimens as a simple and cost-effective 
method to perform analysis of certain genes. We recently established a highly sensitive HPV-PCR method, termed uniplex E6/E7 PCR, which is able to identify the E6 or E7 genes of 39 HPV types, including all HR and pHR types, and some LR HPV types [21].

The aim of this study was to determine the HPV genotype responsible for VaIN using molecular mapping of target lesions in FFPE tissue specimens and the uniplex E6/E7 HPV-PCR test. We attempted to clarify the clinical-pathological classification of the HPV type in VaIN. Primary HPV test screening has been recently launched in some Western countries [22], and VaIN is occasionally found in cervical cancer screening. Therefore, this kind of study may be important for clinical management for VaIN after screening, and for knowledge about the mechanism of vaginal cancer induced by HPV infection. This is the first study to determine HPV types by molecular mapping of VaIN lesions, and may elucidate the HPV types responsible for low-grade lesion (VaIN1), high-grade lesion (VaIN2/3), and VaSCC.

\section{Materials and Methods}

\subsection{Selection of Subjects and Specimens}

In the present study, for convenience the former term, VaIN is used for vaginal squamous intraepithelial lesion (VaSIL), and CIN is used for cervical SIL. One hundred and five patients diagnosed with VaIN and VaSCC, who visited the department of Obstetrics and Gynecology of the Kanazawa Medical University Hospital from 2011 to 2020, were analyzed in this study (Figure 1). All samples were randomized based on the demographic information of all patients. Patients having anal, vulvar, and cervico-vaginal condyloma as the predominant lesion were excluded in this study. All subjects agreed to the use of their cell or histology specimen for this study prior to surgery, according to the protocols approved by the Ethics Committee of Kanazawa Medical University. In total, 12 cases with no or too few histology samples or cell samples and 18 cases with a lack of information were excluded; therefore, 75 patients were evaluated. One pathologist and one surgical pathologist confirmed the diagnosis for each specimen according to the WHO classification [23]. If the diagnosis did not coincide, a final diagnosis was determined via agreement between two pathologists.

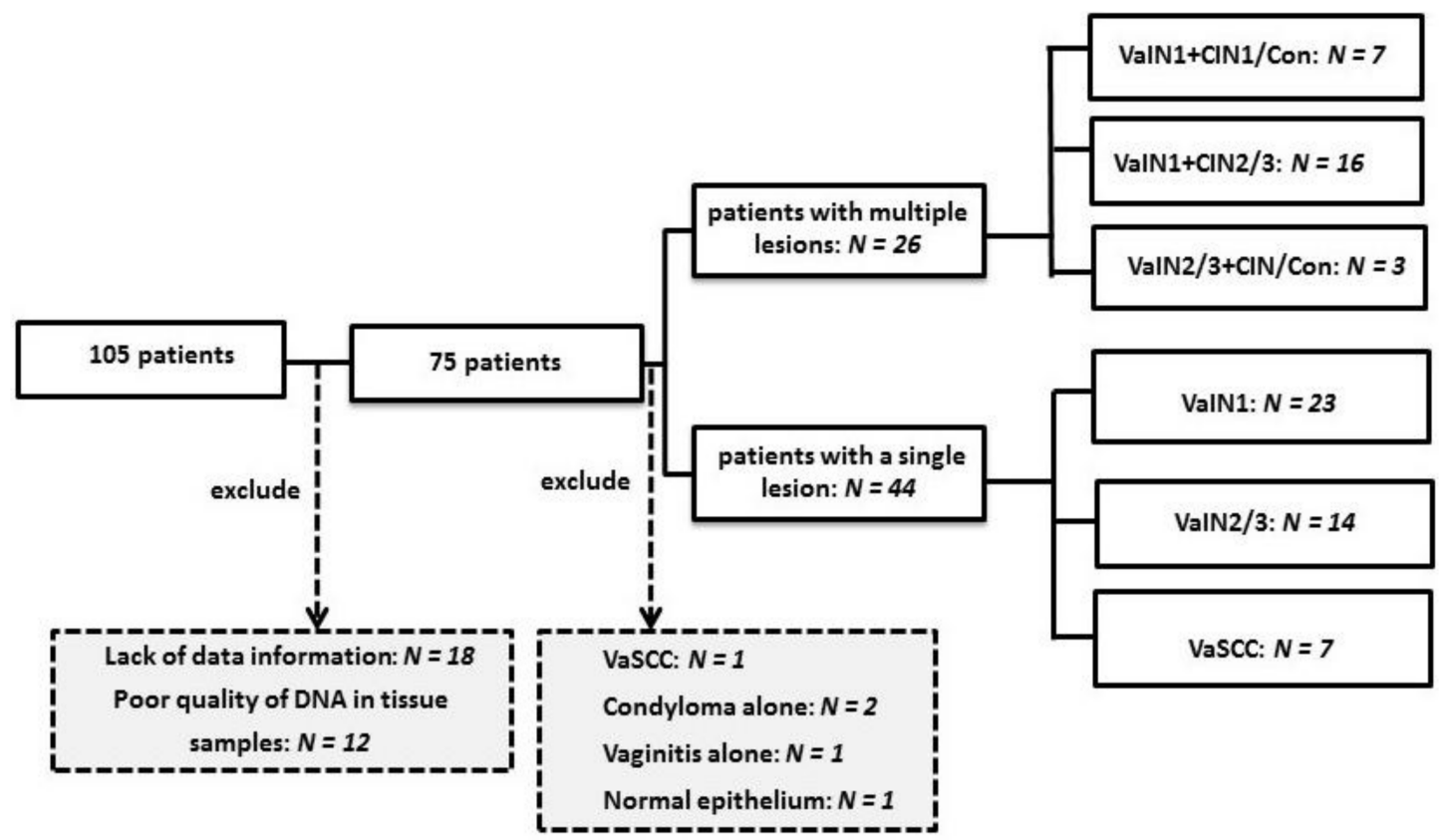

Figure 1. Stratification of patients investigated for vaginal squamous intraepithelial tissue lesions. CIN: cervical intraepithelial neoplasia; Con: condyloma; N: number; VaSCC: vaginal squamous cell carcinoma; VaIN: vaginal intraepithelial neoplasia. 
Two cases with vaginal condyloma, one with vaginitis, one normal epithelium, and one vaginal cancer case were excluded from further analysis (Figure 1). The origin of this cancer case was not clear because the tumor extended from whole vagina to the cervix. The other five cancer cases presented no tumor in the cervix, and two cases had no uteruses due to surgical resection for fibroids when they were younger. Finally, 70 cases were investigated. Among these, 44 cases had one disease, namely, 23 VaIN1, 14 VaIN2/3, and 7 VaSCC cases. The remaining 26 cases were diagnosed with mixed diseases, namely, 23 cases of VaIN1+CIN/condyloma and 3 cases of VaIN2/3+CIN/condyloma. Cervical (predominantly ex-cervix and cervical canal) scraped cell samples were obtained from all subjects, with the exception of five cases, for HPV genotyping with Genoserach-31+4, as described below.

\subsection{HPV Genotyping Using Liquid-Based Cytology (LBC) Specimens with the Genosearch-31+4 Method}

LBC samples were obtained from the cervices of most subjects at their first visit to the out-patient clinic in Kanazawa Medical University Hospital. The samples were stored at $4{ }^{\circ} \mathrm{C}$ until the HPV genotyping test, which was performed within 2 weeks of the collection by a commercial laboratory (LSI medience, Tokyo, Japan). The HPV genotype was determined using the Genosearch-31+4 (GS-31+4) method (Medical \& Biological Laboratories, Co., Ltd., Nagoya, Japan) in the LSI laboratory. This is a new genotyping system based on the PCR-rSSO-Luminex method. This system (GS-31) detects a total of 31 HPV genotypes, including both high-risk (HR), possible high-risk (pHR), and low-risk (LR) HPV types: HPV-6, 11, 16, 18, 26, 31, 33, 35, 39, 42,44, 45, 51, 52, 53, 54, 55, 56, 58, 59, $61,62,66,68,70,71,73,82,84,89$, and 90. An additional four pHR-HPV types (HPV30, 34, 67 , and 69) were also detected with the GS-31+4 system. The GS-31+4 method uses the multiplex primers to amplify different genomic sites of the L1 region on 35 different HPV types, and uses 35 independent probes for detection. It has been confirmed that there is no cross-reaction to other HPV types among these 35 types, and the guaranteed detection limit is 1000 copies of any HPV type in a test [24].

\subsection{Sandwich Cutting Method for FFPE (Formalin Fixed and Paraffin Embedded) Tissue Specimen Analysis}

The FFPE blocks for each case were sectioned according to the following procedure: three slices of $4 \mu \mathrm{M}$ sections were used for histopathological diagnosis after haematoxylin and eosin (H\&E) staining, followed by expression of p16 and Ki67 after immunohistochemical (IHC) staining; five slices of $10 \mu \mathrm{M}$ sections were used for DNA extraction and HPV genotyping; and the final $4 \mu \mathrm{M}$ section was used for histological evaluation with $\mathrm{H} \& \mathrm{E}$ staining to confirm if the target lesion remained. The microtome was cleaned with $70 \%$ alcohol and the blade was replaced for cutting following each tissue block to avoid contamination of HPV in other samples (Figure 2). The tissue slices were placed on individual glass slides that were stored in a refrigerator at $-20^{\circ} \mathrm{C}$ until DNA extraction.

\subsection{Immunohistochemical Staining for p16 and Ki67}

p16 immunohistochemical staining was performed using the CINtec ${ }^{\circledR}$ Histology Kit (Roche mtm laboratories AG, Heidelberg, Germany) according to the manufacturer's instructions. Briefly, the $4 \mu \mathrm{m}$ thick FFPE tissue sections were deparaffinized in xylene and rehydrated in graded alcohols. Antigen retrieval was twice performed in a microwave in Epitope Retrieval Solution (Tris/EDTA, pH 9.0) for $5 \mathrm{~min}$ at $500 \mathrm{~W}$. The slides were cooled to room temperature before equilibration in phosphate buffered saline (PBS), and incubated with Peroxidase-Blocking Reagent to block endogenous peroxidase activity. Subsequently, the slides were incubated with the ready-to-use primary anti-p16 antibody (clone E6H4) for $30 \mathrm{~min}$ at room temperature. The slides were washed in PBS, then incubated with the secondary antibody (Visualization Reagent) for $30 \mathrm{~min}$ at room temperature. The slides were incubated in the Visualization Reagent, which contains a polymer reagent conjugated to horseradish peroxidase (HRP), for $30 \mathrm{~min}$ at room temperature. After washing in 
PBS, incubation with Substrate-Chromogen Solution (1:40 dilution of DAB chromogen in DAB buffer) for 10 min led to p16 brown staining. Mayer's Hematoxylin was used as a counterstain. Slides were washed in deionized water, dehydrated in graded alcohols, and cleared in xylene. A non-aqueous mounting medium was applied to each section before they were mounted with coverslips. For the detection of the Ki67 antigen, we used the monoclonal mouse antibody (MIB-1, code M7240, Dako, Denmark), and the specific procedure was the same as that described above.

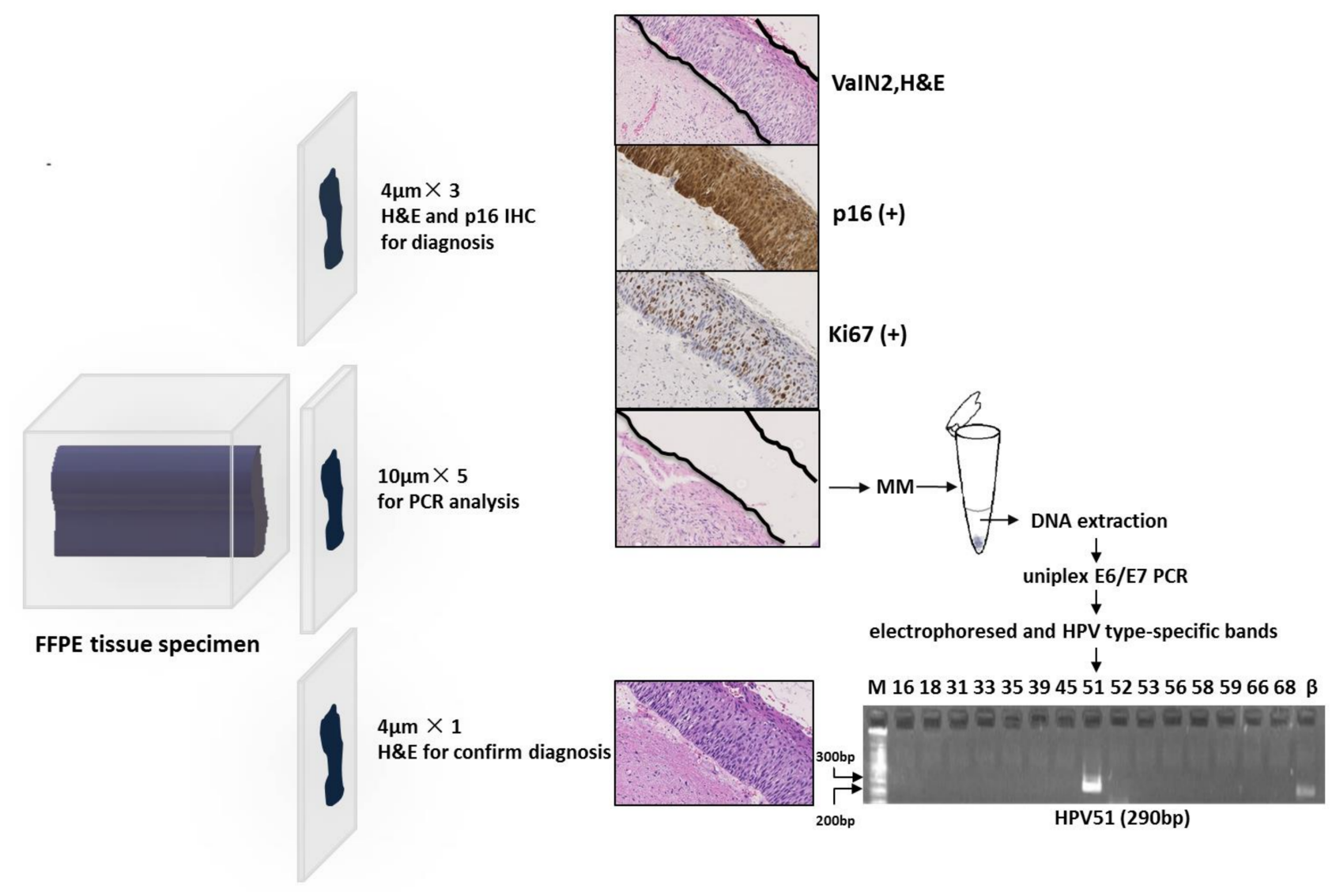

Figure 2. Stratification of patients investigated for vaginal squamous intraepithelial tissue lesions Bp: base pair; FFPE: formalin fixed, paraffin embedded; H\&E: haematoxylin and eosin; IHC: immunohistochemistry; M: marker; MM: manual microdissection; PCR: polymerase chain reaction; $\beta$ : $\beta$-globin.

One normal squamous cell tissue without HPV infection was used as the negative control for p16 and Ki67 staining, and two VaIN3 slides with p16 and Ki67 staining were used as the positive control [25]. p16 was expressed in the cytoplasm and/or nucleus in positive cases, whereas Ki67 was expressed only in the nucleus. If more than $5 \%$ of squamous cells (nuclear and/or cytoplasmic staining) were positive for p16, staining was considered to be positive for p16 expression. The Ki67 index was defined based on the percentage of positive cells: when the index was below $5 \%$ or more than $5 \%$, it was considered to be negative or positive, respectively [25-27].

\subsection{Manual Microdissection and DNA Extraction from Tissue Specimens}

All slides stained with H\&E (the control slides) were reviewed by a surgical pathologist, and the areas of abnormal lesion, and normal squamous or glandular epithelium, were marked on each slide. By viewing the marked areas of the control slide, an abnormal area or a normal tissue on the tissue slides stained with H\&E was independently dissected by hand using a sterilized 21G needle under an inverted microscope (Figure 2). The number of slides used differed in each case according to the size of the target lesion. In the present 
study, three-to-five slides containing VaIN or CIN were needed to test for $39 \mathrm{HPV}$ types in the uniplex E6/E7 PCR system. Each tissue fragment was placed in a $1.5 \mathrm{~mL}$ tube with $50 \mu \mathrm{L}$ of alkaline lysis reagent $(25 \mathrm{mM} \mathrm{NaOH}, 0.2 \mathrm{mM}$ EDTA, pH 12.0), the tube was incubated in a thermo-shaker (Biosan, TS-100, Latvia) at $95{ }^{\circ} \mathrm{C}$ with shaking of 300 revolutions per minute (rpm) for $15 \mathrm{~min}$, and the tube was spun down for $10 \mathrm{~s}$; this procedure was repeated one at $300 \mathrm{rpm}$ and twice at $900 \mathrm{rpm}$. Then, $50 \mu \mathrm{L}$ of acidic neutralizing solution (0.04 mM Tris-HCL, pH 5.0) was added, mixed thoroughly, and centrifuged (12,000 rpm) for one minute. A quantity of $50 \mu \mathrm{L}$ of supernatant was obtained in a new $1.5 \mathrm{~mL}$ tube, and diluted five times with distilled water (DW); $5 \mu \mathrm{L}$ of the solution was used in the uniplex E6-E7 PCR for each HPV type, and the remaining solution was stored in a freezer at $-20{ }^{\circ} \mathrm{C}$ until the subsequent experiment.

\subsection{HPV Genotyping Using FFPE Tissue Specimens}

The uniplex E6/E7 PCR test was designed to amplify E6 and E7 genes of 39 common HPV types using type-specific primer pairs. This assay is able to individually detect 15 LR-HPV types (HPV6, 11, 40, 42, 44, 54, 55, 61, 62, 71, 74, 81, 84, 89, 90); 11 pHR-HPV types (HPV26, 30, 34, 53, 66, 67, 69, 70, 73, 82, 85); 13 HR-HPV types (HPV16, 18, 31, 33, 35, 39, 45, $51,52,56,58,59,68)$; and the beta-globin gene as a positive control [16].

Using $10 \mu \mathrm{L}$ of PCR mixture containing $5.0 \mu \mathrm{L}$ of the sample as a template, $0.5 \mathrm{pM}$ of each HPV type-specific primer, and 5.0 $\mu \mathrm{L}$ of AmpliTaq Gold 360 Master Mix (Applied Biosystem, Foster City, CA), PCR was performed for each HPV type in a T100 ${ }^{\mathrm{TM}}$ Thermal Cycler (Bio-Rad, Hercules, CA) using the following program: $10 \mathrm{~min}$ at $94{ }^{\circ} \mathrm{C} ; 40$ cycles of $30 \mathrm{~s}$ at $94{ }^{\circ} \mathrm{C}, 30 \mathrm{~s}$ at $60^{\circ} \mathrm{C}$, and $30 \mathrm{~s}$ at $72{ }^{\circ} \mathrm{C}$; and a final $5 \mathrm{~min}$ extension step. A quantity of $5.0 \mu \mathrm{L}$ of each reaction solution for each HPV type was applied to a $2.5 \%$ agarose gel (Bio-Rad) and electrophoresed in $1 \times$ TBE buffer for $8 \mathrm{~min}$. HPV type-specific bands were visualized with SYBR ${ }^{\circledR}$ Green I (Takara-bio, Shiga, Japan) staining in a lane under a UV light (Figure 2).

If the beta-globin gene was negative, it was considered to be a poor quality sample or to have contained an insufficient amount of extracted DNA. These samples were observed in 12 of 30 cases, and were excluded from further analysis (Figure 1).

HPV typing results obtained with the uniplex E6-E7 PCR system were compared with the previous results determined by GS-31+4. The latter HPV test could not detect HPV40, 74, 81, and 85, and among these HPV types, only HPV81 was identified in some samples in the present study.

\subsection{Statistical Analysis}

We used Mann-Whitney's U test to undertake analysis of continuous variables (patient's age), and the chi-square test with Yates' correction was used to compare the categorical variables (prevalence of lesions or HPV-positive cases). We used Fisher's exact test when the total sample size was less than 10. A probability value of $p<0.05$ was considered to be statistically significant. All analysis was performed with JSP version 14 (SAS, Tokyo, Japan).

\section{Results}

3.1. Diagnosis and Determination of Vaginal and Cervical Intraepithelial Lesions with P16 and Ki67 Immune-Staining of Tissue Specimens

We used p16 and Ki67 immunohistochemical staining to identify VaIN lesions in tissue specimens using the microdissection procedure. It was shown that $88.2 \%(15 / 17)$ of VaIN2/3 and 28.6\% (14/49) of VaIN1 were positive for p16 expression, whereas this was seen in only $9.5 \%$ of normal glandular or metaplastic cells, and no expression was found in normal squamous epithelium adjacent to VaIN or CIN. Two VaIN2/3 cases negative for p16 were infected with HPV51 (HR-HPV) and HPV42 (LR-HPV). In contrast, most VaIN1 cases $(92.6 \%, 13 / 14)$ positive for p16 expression were infected with HR or pHR-HPV types, although only one p16-positive VaIN1 was infected with an LR-HPV type, namely, 
HPV71. All VaIN2/3 and 81.6\% (40/49) of VaIN1 lesions were positive for Ki67. Of six VaIN1 lesions found to be negative for HPV, four lesions were found to be positive for Ki67 but negative for $\mathrm{p} 16$.

\subsection{The Prevalence of HPV Infection in Cases with Vaginal Intraepithelial Neoplasia (VaIN)}

We examined the prevalence of HPV infection in 63 eligible selected cases with VaIN and seven cases with VaSCC. All of these subjects visited our out-patient clinic to undertake further examination for abnormal Pap test worse than atypical squamous cells undetermined significance (ASC-US). All subjects were classified into two groups: the single lesion group, comprising cases with VaIN or VaSCC alone; and the multiple lesions group, comprising cases with VaIN combined with CIN or condyloma. The former group was composed of $23 \mathrm{VaIN1}, 14 \mathrm{VaIN} 2 / 3$, and $7 \mathrm{VaSCC}$ cases, whereas the latter group comprised $7 \mathrm{VaIN} 1+\mathrm{CIN} 1$ or condyloma, $16 \mathrm{VaIN} 1+\mathrm{CIN} 2 / 3$, and $3 \mathrm{VaIN} 2 / 3+\mathrm{CIN} 1$ or condyloma (Figure 1). Therefore, 46 cases were diagnosed with VaIN1 and 17 cases with VaIN2/3 (Figure 1).

The comparison of the ages of cases (Table 1) showed no statistical difference between the cases with VaIN1 and those with VaIN2/3, and between cases with a single lesion and those with multiple lesions (Fisher's test, $p>0.1$ ). Seven cases with VaSCC were older (av. $81.7 \mathrm{y}$ ) than the other VaIN cases (av. $45.5 \mathrm{y}$ ) (Mann-Whitney's U test, $p=0.013$ ).

HPV genotyping in tissue specimens was performed in all the subjects, whereas HPV typing in scraped cell samples was performed in 36 cases with a single VaIN lesion, 24 cases with multiple lesions, and 3 VaSCC cases. Regarding tissue results, overall HPV prevalence in all subjects was 95.7\% (Table 1).

Among the single lesion group, the prevalence of any HPV types did not differ between VaIN1 and VaIN2/3 ( $p>0.1)$. As for the multiple lesions group, the prevalence of HPV types was calculated separately between VaIN and CIN (Condyloma) groups (Table 2).

\subsection{Comparison of the Prevalence of HPV Genotypes in Tissue Specimens and in Cervicovaginal Scraped Cells}

HPV genotyping results in the single lesion group are shown in Table 3 , and those in multiple lesions are shown in Table 4. First, we analyzed HPV typing results from cervicovaginal cell samples and tissue samples in 39 eligible cases with a single lesion. It was demonstrated that multiple HPV types were detected in 53.8\% (21/39) of these cell samples; in contrast, multiple HPV types in tissue samples were identified in only two cases (Table 3).

When we compared HPV genotype results in tissue and scraped cell samples in the single lesion group (Table 3), eight cases were excluded, because five cases had no HPV data in the scraped cell samples, and three further cases could not be evaluated because HPV81 identified in the tissue specimens was not detectable by another HPV assay (GS-31+4) using the cell sample. This comparison revealed that complete and partial discordance for HPV genotypes between cell and tissue samples was observed in 9 cases $(25.0 \%, 9 / 36)$ and 15 cases $(41.7 \%, 15 / 36)$, respectively. Therefore, $66.7 \%(24 / 36)$ of these cases showed a discrepancy in the HPV genotype between cell and tissue samples. Moreover, 15 cases $(41.7 \%, 15 / 36)$ that were positive for certain HR-HPV types detected in cell samples were not present in the tissue specimen (Table 3). About $40 \%$ of these preclinical HR-HPV infections were observed in cases with a single VaIN. This was more common in the case of VaIN1 $(11 / 19,57.9 \%)$ than in VaIN2/3 $(2 / 14,14.3 \%)(p=0.030)$.

The same analysis was performed for 24 eligible cases with multiple lesions who undertook HPV testing using both cell and tissue samples. Various HPV types that had been detected in cell samples were not present in $70.8 \%(17 / 24)$ of tissue specimens. Regarding HR-HPV type, these preclinical infections were recognized in $54.2 \%(13 / 24)$ of the multiple lesion group. Conversely, the opposite result, in which the HPV genotype was not identified in cell samples but was positive in tissues, was seen in two cases (Cases \#34, 48). 
Table 1. Age of patients, histological diagnosis and HPV infection by E6/E7 PCR using tissue specimens.

\begin{tabular}{|c|c|c|c|c|c|c|c|c|c|}
\hline \multirow[b]{2}{*}{ Diagnosis } & \multicolumn{3}{|c|}{ Cases with a Single Lesion } & \multicolumn{6}{|c|}{ Cases with Multiple Lesions } \\
\hline & VaIN1 & VaIN2/3 & & VaSCC & & VaIN1+CIN1/Con & VaIN1+CIN2/3 & VaIN2/3+CIN/Con & Total \\
\hline & $N=23$ & $N=14$ & $p^{1}$ & $N=7$ & $p^{2}$ & $N=7$ & $N=16$ & $N=3$ & $N=70$ \\
\hline HPV prevalence $(\%)$ & $95.7(22 / 23)$ & $100(14 / 14)$ & $>0.1$ & $85.7(6 / 7)$ & & $85.6(6 / 7)$ & $100(16 / 16)$ & $100(3 / 3)$ & $95.7(67 / 70)$ \\
\hline HR-HPV (\%) * & $17.4(4 / 23)$ & $85.7(12 / 14)$ & & $57.1(4 / 17)$ & & $28.6(2 / 7)$ & $93.8(15 / 16)$ & $66.7(2 / 3)$ & $55.7(39 / 70)$ \\
\hline Possible HR-HPV (\%) * & $34.8(8 / 23)$ & $0(0 / 14)$ & & $28.6(2 / 7)$ & & $57.1(4 / 7)$ & $43.8(7 / 16)$ & $33.3(1 / 3)$ & $31.4(20 / 70)$ \\
\hline LR-HPV (\%) * & $43.5(10 / 23)$ & $14.3(2 / 14)$ & & $0(0 / 7)$ & & $42.9(3 / 7)$ & $25.0(4 / 16)$ & $66.7(2 / 3)$ & $30.0(21 / 70)$ \\
\hline Single HPV type (\%) & $95.7(22 / 23)$ & $78.6(11 / 14)$ & & $85.7(6 / 7)$ & & $14.3(1 / 7)$ & $25.0(4 / 16)$ & $0(0 / 3)$ & $62.9(38 / 70)$ \\
\hline Multiple HPV types (\%) & $0(0 / 23)$ & $21.4(3 / 14)$ & & $0(0 / 7)$ & & $71.4(5 / 7)$ & $75.0(12 / 16)$ & $100(3 / 3)$ & $32.9(23 / 70)$ \\
\hline
\end{tabular}

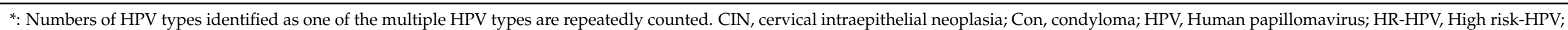


and VaIN2/3 cases. $p^{2}$ value means comparing the age between all VaIN and VaSCC cases.

Table 2. Age of patients, histological diagnosis and HPV infection by E6/E7 PCR using tissue specimens.

\begin{tabular}{|c|c|c|c|c|c|c|c|c|c|}
\hline \multirow[b]{2}{*}{ Diagnosis } & \multicolumn{3}{|c|}{ Cases with a Single Lesion } & \multicolumn{6}{|c|}{ Cases with Multiple Lesions } \\
\hline & VaIN1 & VaIN2/3 & & VaSCC & & VaIN1+CIN1/Con & VaIN1+CIN2/3 & VaIN2/3+CIN/Con & Total \\
\hline & $N=23$ & $N=14$ & $p^{1}$ & $N=7$ & $p^{2}$ & $N=7$ & $N=16$ & $N=3$ & $N=70$ \\
\hline HPV prevalence (\%) & $95.7(22 / 23)$ & $100(14 / 14)$ & $>0.1$ & $85.7(6 / 7)$ & & $85.6(6 / 7)$ & $100(16 / 16)$ & $100(3 / 3)$ & $95.7(67 / 70)$ \\
\hline \multirow[t]{3}{*}{ HR-HPV (\%) * } & $17.4(4 / 23)$ & $85.7(12 / 14)$ & & $57.1(4 / 17)$ & & $28.6(2 / 7)$ & $93.8(15 / 16)$ & $66.7(2 / 3)$ & $55.7(39 / 70)$ \\
\hline & & & & & & $\begin{array}{c}\text { VaIN1 } \\
\text { CIN1/Con }\end{array}$ & $\begin{array}{c}\text { VaIN1 } \\
\text { CIN2/3 }\end{array}$ & $\begin{array}{l}\text { VaIN2/3 } \\
\text { CIN/Con }\end{array}$ & \\
\hline & & & & & & $\begin{array}{c}0(0 / 7) \\
28.6(2 / 7)\end{array}$ & $\begin{array}{c}6.25(1 / 16) \\
87.5(14 / 16)\end{array}$ & $\begin{array}{l}66.7(2 / 3) \\
33.3(1 / 3)\end{array}$ & \\
\hline
\end{tabular}


Table 2. Cont.

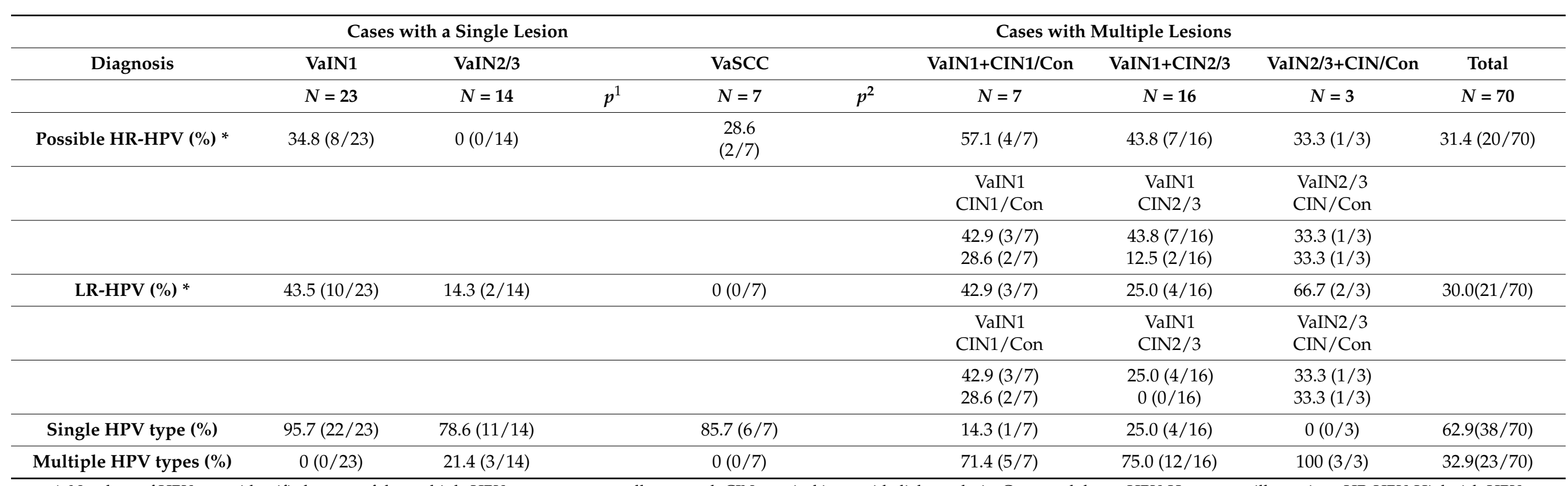

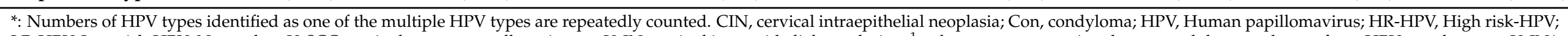

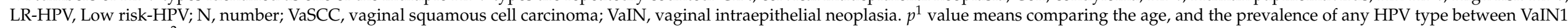

and VaIN2/3 cases. $p^{2}$ value means comparing the age between all VaIN and VaSCC cases. 
Table 3. Comparison of HPV type results determined by tissue or scraped cell samples in cases with a single lesion.

\begin{tabular}{|c|c|c|c|c|c|}
\hline Case & VaIN & HPV Type in Tissue & HPV Type in Scraped & Discordance in HPV Type & HR-HPV May Exist \\
\hline No. & & by Uniplex E6/E7 PCR & Cells by GS31+4 & in Tissue and Cell Samples & in Preclinical Lesions \\
\hline 1 & VaIN1 & HPV81* & Negative & NE & \\
\hline 4 & VaIN1 & HPV84 & HPV51,58 & Completely & 51,58 \\
\hline 7 & VaIN1 & HPV90 & HPV16,90 & Partly & 16 \\
\hline 8 & VaIN1 & HPV81* & HPV51,66,89,42 & $\mathrm{NE}$ & 51 \\
\hline 11 & VaIN1 & HPV66 & HPV66,89 & Partly & \\
\hline 16 & VaIN1 & Negative & HPV31 & Completely & 31 \\
\hline 18 & VaIN1 & HPV16 & HPV31,82 & Completely & 31 \\
\hline 27 & VaIN1 & HPV53 & HPV53 & No & \\
\hline 28 & VaIN1 & HPV90 & HPV55,61 & Completely & \\
\hline 29 & VaIN1 & HPV53 & HPV53,62 & Partly & \\
\hline 30 & VaIN1 & HPV68 & HPV52 & Completely & 52 \\
\hline 36 & VaIN1 & HPV66 & HPV66 & $\mathrm{No}$ & \\
\hline 39 & VaIN1 & HPV61 & HPV39,61 & Partly & 39 \\
\hline 40 & VaIN1 & HPV61 & HPV31,61 & Partly & 31 \\
\hline 45 & VaIN1 & HPV58 & HPV58 & No & \\
\hline 50 & VaIN1 & HPV67 & HPV67 & No & \\
\hline 52 & VaIN1 & HPV73 & HPV73 & No & \\
\hline 53 & VaIN1 & HPV90 & Negative & Completely & \\
\hline 55 & VaIN1 & HPV56 & HPV52,56 & Partly & 52 \\
\hline 59 & VaIN1 & HPV71 & Negative & Completely & \\
\hline 62 & VaIN1 & HPV34 & NE & $\mathrm{NE}$ & \\
\hline
\end{tabular}


Table 3. Cont

\begin{tabular}{|c|c|c|c|c|c|}
\hline Case & VaIN & HPV Type in Tissue & HPV Type in Scraped & Discordance in HPV Type & HR-HPV May Exist \\
\hline No. & & by Uniplex E6/E7 PCR & Cells by GS31+4 & in Tissue and Cell Samples & in Preclinical Lesions \\
\hline 5 & VaIN2/3 & HPV52 & HPV52 & No & \\
\hline 14 & VaIN2/3 & HPV16 & HPV16,84,89,90 & Partly & \\
\hline 17 & VaIN2/3 & HPV52 & HPV52,84 & Partly & \\
\hline 25 & VaIN2/3 & HPV42 & HPV6,42 & Partly & \\
\hline 57 & VaIN2/3 & HPV42 & HPV42 & No & \\
\hline 6 & VaIN2/3 & HPV16 & HPV16,89 & Partly & \\
\hline 19 & VaIN2/3+VaIN1 & HPV58+HPV31 & HPV31,54,58,90 & Partly & \\
\hline 23 & VaIN2/3+VaIN1 & HPV51+HPV90 & HPV39,51,90 & Partly & 39 \\
\hline 41 & VaIN2/3 & HPV16 & HPV16 & No & \\
\hline 42 & VaIN2/3 & HPV16 & HPV68 & Completely & \\
\hline 44 & VaIN2/3 & HPV56 & HPV56 & No & \\
\hline 54 & VaIN2/3 & HPV58 & HPV39,58 & Partly & 39 \\
\hline 64 & VaSCC & HPV16 & HPV16 & No & \\
\hline 65 & VaSCC & HPV45 & NE & $\mathrm{NE}$ & \\
\hline 66 & VaSCC & HPV58 & HPV16,52,58 & Partly & 16,52 \\
\hline 67 & VaSCC & HPV68 & NE & NE & \\
\hline 68 & VaSCC & HPV53 & NE & $\mathrm{NE}$ & \\
\hline 69 & VaSCC & HPV67 & HPV6,52 & Completely & 52 \\
\hline 70 & VaSCC & Negative & NE & NE & \\
\hline
\end{tabular}

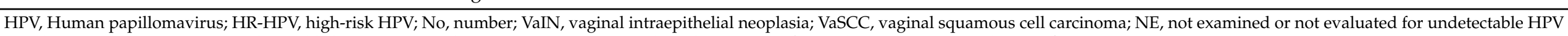
type by GS31+4; ${ }^{*}$, HPV81 is not detectable by GS31+4; $p$ value means comparing preclinical HR-HPV infection between VaIN1 and VaIN2/3 cases. 
Table 4. Compariosn of HPV type results determined by tissue or scraped cell-samples in cases with multiple lesions.

\begin{tabular}{|c|c|c|c|c|c|c|c|}
\hline VaIN & HPV Type in Tissue & Acompanied & HPV Type in Tissue & $\begin{array}{c}\text { Discordance for } \\
\text { HPV Type }\end{array}$ & HPV Type in Cells & $\begin{array}{l}\text { Discordance for } \\
\text { HPV Type }\end{array}$ & HR-HPV May Exis \\
\hline Lesion & by E6/E7 PCR & Lesion & by E6/E7 PCR & VAIN vs. CIN/Con & by GS-31+4 & Tissue vs. Cells & $\begin{array}{l}\text { in Preclinical } \\
\text { Lesions }\end{array}$ \\
\hline VaIN1 & HPV53 & CIN3 & HPV52 & Yes & HPV16,39,52,90 & Partly & 16,39 \\
\hline VaIN1 & Negative & CIN2 & HPV18 & Yes & HPV18 & No & \\
\hline VaIN1 & Negative & CIN2 & HPV52 & Yes & HPV16,52 & Partly & 16 \\
\hline VaIN1 & Negative & CIN1 & Negative & No & Negative & No & \\
\hline VaIN1 & Negative & CIN3 & HPV51 & Yes & HPV51 & No & \\
\hline VaIN1 & HPV42 & CIN2 & HPV52 & Yes & HPV42,52 & No & \\
\hline VaIN1 & HPV53 & CIN1 & HPV68 & Yes & HPV52,53,68 & Partly & 52 \\
\hline VaIN1 & HPV42 & CIN2 & HPV58 & Yes & HPV31, 51 & Completely & 31,51 \\
\hline VaIN1 & HPV67 & CIN2 & HPV52 & Yes & HPV52 & Partly & \\
\hline VaIN1 & HPV81 & CIN3 & HPV16 & Yes & HPV16,35,51,53 & $\mathrm{NE}$ & 35,51 \\
\hline VaIN1 & HPV71 & CIN1 & HPV84 & Yes & HPV11,16,53,54,62,84 & Partly & 16 \\
\hline VaIN1 & HPV52 & CIN2 & HPV66 & Yes & HPV16,52,66 & Partly & 16 \\
\hline VaIN1+metaplasia & HPV66+HPV74 & CIN1 & HPV53 & Yes & HPV42,52,53 & Partly & 52 \\
\hline VaIN1 & HPV53 & Condyloma & HPV11 & Yes & HPV56,53,73 & Partly & 56 \\
\hline VaIN1 & HPV90 & CIN1+CIN1 & HPV52+HPV68 & Yes & HPV52,68 & Partly & \\
\hline VaIN1 & HPV34 & CIN2 & HPV39 & Yes & $\mathrm{NE}$ & $\mathrm{NE}$ & \\
\hline VaIN1 & HPV34 & CIN3 & HPV52 & Yes & HPV34,52 & No & \\
\hline VaIN1 & HPV53 & CIN2 & HPV39 & Yes & HPV16,42,89 & Completely & 16 \\
\hline VaIN1 & HPV84 & CIN3 & HPV52 & Yes & $\mathrm{NE}$ & $\mathrm{NE}$ & \\
\hline
\end{tabular}


Table 4. Cont.

\begin{tabular}{|c|c|c|c|c|c|c|c|}
\hline VaIN & HPV Type in Tissue & Acompanied & HPV Type in Tissue & $\begin{array}{l}\text { Discordance for } \\
\text { HPV Type }\end{array}$ & HPV Type in Cells & $\begin{array}{l}\text { Discordance for } \\
\text { HPV Type }\end{array}$ & HR-HPV May Exist \\
\hline Lesion & by E6/E7 PCR & Lesion & by E6/E7 PCR & VAIN vs. CIN/Con & by GS-31+4 & Tissue vs. Cells & $\begin{array}{c}\text { in Preclinical } \\
\text { Lesions }\end{array}$ \\
\hline VaIN1 & Negative & CIN2 & HPV51 & Yes & HPV51 & No & \\
\hline VaIN1 & HPV66 & CIN3+CIN1 & HPV16+HPV66 & Yes & HPV16,52,59,53,66,82 & Partly & 52,59 \\
\hline VaIN2/3 & HPV66 & CIN1 & HPV67 & Yes & HPV82 & Completely & \\
\hline VaIN2/3+VaIN1 & HPV51+HPV71 & CIN2 & HPV51 & Yes & HPV51,71,68 & Partly & 68 \\
\hline
\end{tabular}

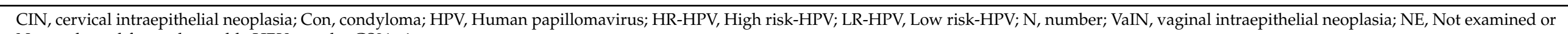
Not evaluated for undetectable HPV type by GS31+4. 
It is surprising that the frequency of the preclinical HPV infection did not differ between the single lesion and multiple lesion groups (Fisher's test, $p>0.1$ ). In all subjects, $68.3 \%(41 / 60)$ had preclinical infection with any HPV type, and 46.7\% (28/60) were found to have a preclinical HR-HPV-type infection. Moreover, HPV genotypes in VaIN and CIN lesions differed in $92.3 \%(24 / 26)$ of cases with multiple lesions (Table 4$)$. This suggests that the vaginal and cervical lesions developed independently in most cases.

\subsection{Determination of HPV Genotypes Responsible for VaSCC, VaIN, and CIN by Molecular Mapping}

Epithelial tissues including target lesions were manually dissected for HPV genotyping in all cases, and DNA samples were obtained from 99 sections and evaluated for HPV genotypes. It was revealed that 57 of $63(90.5 \%)$ VaIN tissues were positive for HPV (Tables 3 and 4). One VaIN tissue in the single lesion group, and five VaIN tissues in the multiple lesion group, were found to be negative for any HPV type (Tables 3 and 4 ). Two cases in the single lesion group (Cases \#19,23) and four cases of the multiple lesion group (Cases \#32, 46, 48, 63) showed multiple HPV type infections in one tissue specimen (Tables 3 and 4). We re-evaluated the histology of these six specimens that showed multiple HPV type infections, and found that morphologically different areas existed in some slides for each case. Two or three separable lesions were cut out by manual dissection under the guidance of counter-staining with hematoxylin and expression of p16 or Ki67, as described in the Materials and Methods section (Figure 2). This revealed that different HPV types were identified in each of VaIN2/3 and VaIN1 lesions in Case\#19, Case \#23, Case \#32; VaIN1 and metaplasia in Case \#46; CIN2/3 and CIN1 lesions in Case\#63; and two CIN1 lesions in Case \#48 (Tables 3 and 4).

In 49 VaIN1 lesions, 17 HPV types were detected in 43 lesions $(87.8 \%, 43 / 49)$, including HR-HPV in 6 lesions, pHR-HPV in 18 lesions, and LR-HPV in 19 lesions. The most common type was HPV53, which was found in six VaIN1 lesions, followed by HPV66/81/90 in five lesions, HPV34/67/71 in three lesions, HPV42/61/84 in two lesions, and HPV16/31/52/56/58/68/73 in one lesion. In higher-grade lesions, eight HPV types were identified in VaIN2/3, whereas six HPV types were found in VaSCC. The most common type in VaIN2/3 was HPV16 (HR-HPV) $(35.3 \%, 6 / 17)$, followed by HPV51/52/58 (HR-HPV) (11.8\%, 2/17), HPV42 (LR-HPV) (11.8\%, 2/17), and HPV18/56 (HR-HPV) and HPV66 (pHR-HPV) in one case each. In contrast, HPV16/45/58/68 (HR-HPV) and HPV53/67 (pHR-HPV) were identified as one HPV type in each case of VaSCC, and a negative result was detected in one case. Among the 13 internationally defined HR-HPV types, four HPV types (HPV33, 35, 39 and 59) were not detected in any VaIN lesions in the present study, although HPV39 was identified in two CIN2 cases (Tables 4 and 5).

The prevalence of HR-HPV types was more common in VaIN2/3 (85.7\%) than in VaIN1 $(17.4 \%)(p<0.001)$, whereas possible HR (pHR) HPV types were more common in VaIN1 $(34.8 \%)$ than VaIN2/3 (5.9\%) $(p=0.015)$. The LR-HPV type was marginally more prevalent in VaIN1 than VaIN2/3 $(p=0.084)$ (Table 5). 
Table 5. Identified HPV type in a histological tissue of VaIN, VaSCC and CIN.

\begin{tabular}{|c|c|c|c|c|c|c|c|c|c|}
\hline & HDY T & VaIN1 & VaIN2/3 & & VaSCC & Total & CIN1 & CIN2/3 & Total \\
\hline & nov rype & $N=49 *$ & $N=17$ & $p$ & $N=7$ & No. & $N=10$ & $N=16$ & No. \\
\hline \multirow{9}{*}{ High-risk HPV } & HPV16 & 1 & 6 & & 1 & 8 & & 2 & 2 \\
\hline & HPV31 & 1 & & & & 1 & & & \\
\hline & HPV39 & & & & & & & 2 & 2 \\
\hline & HPV45 & & & & 1 & 1 & & & \\
\hline & HPV51 & & 2 & & & 2 & & 3 & 3 \\
\hline & HPV52 & 1 & 2 & & & 3 & 1 & 6 & 7 \\
\hline & HPV56 & 1 & 1 & & & 2 & & & \\
\hline & HPV58 & 1 & 2 & & 1 & 4 & & 1 & 1 \\
\hline & HPV68 & 1 & & & 1 & 2 & 2 & & 2 \\
\hline \multirow{4}{*}{ Possibly high-risk HPV } & HPV34 & 3 & & & & 3 & & & \\
\hline & HPV53 & 6 & & & 1 & 7 & 1 & & 1 \\
\hline & HPV67 & 3 & & & 1 & 4 & 2 & & 2 \\
\hline & HPV73 & 1 & & & & 1 & & & \\
\hline \multirow{7}{*}{ Low-risk HPV } & HPV42 & 2 & 2 & & & 4 & & & \\
\hline & HPV61 & 2 & & & & 2 & & & \\
\hline & HPV71 & 3 & & & & 3 & & & \\
\hline & HPV81 & 5 & & & & 5 & & & \\
\hline & HPV84 & 2 & & & & 2 & 1 & & 1 \\
\hline & HPV90 & 5 & & & & 5 & 1 & & 1 \\
\hline & Negative & 6 & 0 & & 1 & 7 & 1 & 0 & 1 \\
\hline
\end{tabular}


Table 5. Cont

\begin{tabular}{|c|c|c|c|c|c|c|c|c|}
\hline HPV Type & VaIN1 & VaIN2/3 & & VaSCC & Total & CIN1 & CIN2/3 & Total \\
\hline & $N=49 *$ & $N=17$ & $p$ & $N=7$ & No. & $N=10$ & $N=16$ & No. \\
\hline Any HPV type & $43(87.8 \%)$ & $17(100 \%)$ & & $6(85.7 \%)$ & $66(90.4 \%)$ & $9(90.0 \%)$ & $16(100 \%)$ & $25(96.1 \%)$ \\
\hline Possibly HR-HPV type & 18 & 1 & 0.015 & 2 & 21 & 4 & 1 & 5 \\
\hline LR-HPV type & 19 & 2 & 0.084 & 0 & 21 & 2 & 0 & 2 \\
\hline Total & 49 & 17 & & 7 & 73 & 10 & 16 & 26 \\
\hline
\end{tabular}

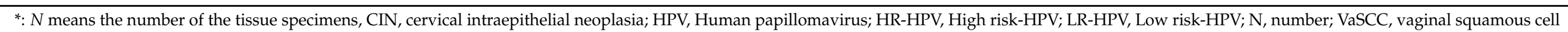

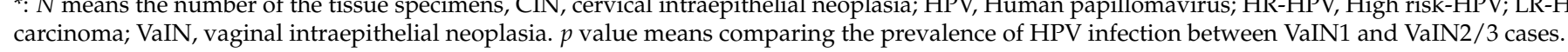




\section{Discussion}

The present study showed the prevalence of HPV was 95.7\% in low-grade vaginal squamous intraepithelial lesions (VaIN1s), 100\% in high-grade lesions (VaIN2 and VaIN3), and $85.7 \%$ in cancer (VaSCC). Different studies have shown different prevalence results of HPV in VaINs. A meta-analysis showed that the overall prevalence of HPV in VaIN ( $n=1374)$ was $85.2 \%$, with the prevalence of each study ranging from $20 \%$ to $100 \%$ [28]. Frega et al. [29] reported that the HPV positive rate in VaIN cases is $100 \%$; however, 40 of 44 of these cases had a past history of cervical carcinoma. In contrast, Tsimplaki et al. [30] reported that the prevalence of HPV was $56 \%$ in VaIN cases who had no history of other HPV-associated malignancies. This difference is likely to be due to selection bias or different HPV detection methods. The former report selected a higher risk group because most cases have a past history of cervical cancer. To examine HPV genotypes in VaIN cases, this report examined scraped cell samples, and found that all samples were positive for HPV16 (80\%) or HPV18 (20\%). Using whole FFPE tissue samples of VaIN, Tsimplaki et al. [30] identified $24 \mathrm{HPV}$ types, including $13 \mathrm{HR}-\mathrm{HPV}$ types, 5 pHR-HPV types, and 6 LR-HPV types. Chao et al. [31] investigated 450 VaIN cases for HPV genotyping using FFPE whole tissue specimens, and testing was able to detect $38 \mathrm{HPV}$ types. This report showed that $41.8 \%$ of VaIN1 and $58.2 \%$ of VaIN2/3 were positive for HPV. Although this study was similar to the present study regarding the sample and HPV detection procedure, the present study showed a higher prevalence of HPV, which was more than $96 \%$. This difference might be due to the difference in the method used. The studies of Tsimplaki et al. and Chao et al. used whole FFPE tissue specimens, whereas the present study used an epithelial tissue of the target lesion for HPV genotyping. Quint $W$ et al. [32] already demonstrated that 100\% of anal intraepithelial neoplasia (AIN) samples, which were obtained via laser capture microdissection, were positive for HPV using the SPF10-PCR method. The present study may be the first to demonstrate that the manual microdissection of VaIN or CIN epithelia from the FFPE tissue specimen is as effective as the LCM procedure in the other studies [20]. In our preliminary experiment, HPV and the beta-globin gene, as the internal control, were not detected in some of the cervical cancer specimens that were taken more than 10 years ago. Furthermore, in addition to the quality of the sample, molecular mapping of the target lesion is also crucial for successful HPV genotyping analysis of a fragment as small as that of VaIN. Therefore, the difference in HPV prevalence in VaIN reported in different studies may be due to sample selection, and the size and quality of the tissue sample.

In the present study, $63 \mathrm{VaIN}$ cases were diagnosed randomly in women who were referred to our out-patient clinic due to an abnormal Pap test result or for follow up for CIN1, 2. This study used a highly sensitive HPV test, uniplex E6/E7 PCR, which is able to detect $39 \mathrm{HPV}$ types, including all HR- and pHR-HPV types, and some LR-HPV types. As mentioned above, all VaIN2/3 lesions were positive for HPV, suggesting that the uniplex E6/E7 PCR method may encompass almost all of the HPV genotypes that might be responsible for VaIN2/3.

Histological evaluation demonstrated 37 cases (56.6\%) with VaIN alone, and $26(40.0 \%)$ cases with VaIN and CIN or condyloma. In the latter cases, 24 cases $(92.3 \%)$ were infected with different HPV types in each lesion of VaIN and CIN; this result was not expected. This suggests that VaIN and CIN were independently developed. Moreover, HPV genotyping results from the cell samples showed additional HPV types that were not identified. Okodo $M$ et al. [21] confirmed that HPV types determined using the uniplex E6/E7 PCR method, which was used in the present study, were highly consistent with the results achieved from the GS-31+4 test using the same cell samples. Therefore, the discrepancy in the HPV results between different samples was not due to the difference in the assay used. The present analysis using the microdissection procedure clearly demonstrated a single HPV type in one lesion. The two assays used in the present study theoretically provide results that are specific to the HPV type. Therefore, the above-mentioned additional HPV types that were detected only in cell samples may be derived from preclinical infections somewhere in the cervix or vagina. If this hypothesis is correct, these preclinical infections existed in 
$69 \%$ of VaIN cases, and those with HR-HPV types were seen in $45 \%$ of cases. Van Der Marel J et al. [19] also reported that, based on cytology samples, $62 \%$ of the CIN3 women had infections of multiple HPV types, whereas the cytological HPV genotypes were not detected in the corresponding CIN3 lesions. These findings indicate that HPV genotype results determined from scraped cell samples are not always derived from a target lesion defined by colposcopy. For this reason, only a single HPV type result was adopted as the type responsible for the lesion in our previous studies [16,18].

In patients with multiple lesions, 92.3\% (24/26) had completely different HPV types in the lesions of CIN and VaIN (Table 4). In these cases, 12 cases had different grades of lesions in different areas; VaIN2/3+CIN1 or CIN2/3+VaIN1, and HR-HPV, were found in all high-grade lesions, and LR- or PHR-HPV were found in seven low-grade lesions in different sites, such as VaIN1+CIN1 or VaIN1+congdyloma. This can also indicate that high-grade lesions are caused by HR-HPV types, whereas low-grade lesions are caused by low-risk or $\mathrm{pHR}$-HPV types. This tendency was statistically relevant. High-grade lesions, including $17 \mathrm{VaIN} 2 / 3$ and 7 VaSCC tissue specimens, were infected with HR HPV typesHPV16, 52, 58 (Alpha-9), 18, 45 (Alpha-7), 51 (Alpha-5), and 56 (Alpha-6); two lesions with LR-HPV type 42 (Alpha-1); and one lesion with pHR-HPV type 53, 66 (Alpha-6), and 67 (Alpha-9). Okodo $\mathrm{M}$ et al. [21] reported that no LR-HPV types were identified as a single-type infection in CIN2/3 and cancer; this is consistent with the present results, in which no low-risk types were identified in 14 specimens of CIN2/3. Similarly, 14 VaIN2/3 and 4 VaSCC lesions were positive for HR-HPV, 1 VaIN2/3 and 2 VaSCC for $\mathrm{pHR}-\mathrm{HPV}$ (HPV53, 66, 67), and 2 VaIN2/3 for HPV42. In our previous study, HPV52, 16, 51, 56, and 42 were identified as a single type in VaIN2/3 [16]. Summarizing both sets of data, the most common species was Alpha-9 (HPV16, 52, 58, 67) (13 lesions), followed by Alpha-6 (HPV53, 56, 66) (3 lesions), Alpha-7 (HPV18, 45, 68) (3 lesions), Alpha-5 (HPV51) (2 lesions), and Alpha-1 (HPV42) (2 lesions). These results suggest that almost all were HR- or pHR-HPV types. Some studies suggest that some pHR-HPV types, such as HPV26, 66, 67, 69, 73, and 82, are likely to be oncogenic [8-10] More surprisingly, one LR-HPV type (HPV42) (Alpha-1) was detected in two cases of VaIN2/3. Guimera et al. [33] demonstrated that HPV42 is found in a typical squamous cell carcinoma of the cervix. Further study is needed to confirm the oncogenicity of this type.

We used p16 and Ki67 immunohistochemical staining to identify VaIN lesions in tissue specimens using the microdissection procedure. It was shown that most of VaIN2/3 $(15 / 17)$ and $28.6 \%(14 / 49)$ of VaIN1 were positive for p16 expression, and no expression was found in normal squamous epithelium adjacent to VaIN or CIN. Two VaIN2/3 lesions negative for p16 were infected with HPV51 (HR-HPV) and HPV42 (LR-HPV), whereas the other cases that were positive for p16 were infected with HR or pHR HPV types. Moreover, most VaIN1 cases $(92.6 \%$; $13 / 14)$ positive for p16 expression were infected with HR- or pHR-HPV types, although only one p16-positive VaIN1 was infected with HPV71 (LR-HPV). In contrast, all VaIN2/3 and most VaIN1 (81.6\%; 40/49) were positive for Ki67. Despite some exceptions, Ki67 expression is useful to discriminate any VaIN lesions from normal squamous epithelium, and p16 expression is associated with HR-HPV or pHR-HPV infection, regardless of the grades of vaginal lesion. Similar conclusions were reported by Chao et al. [31]. In the present study, among the six VaIN1 lesions that were found to be negative for HPV, four HPV-negative lesions were positive for Ki67 expression, but negative for p16, suggesting that these lesions might be infected with LR-HPV types that are unable to be detected by the present HPV test. Low viral load and DNA fragmentation are likely to be the cause of these negative results.

The present study showed that HR-HPV is more common in VaIN2/3, whereas $\mathrm{pHR}$ and LR-HPV are more common in VaIN1. VaIN1 were positive for any type of HPV. Castle et al. reported that LR-HPV types belonging to Alpha-3 and -15 are more prevalent in the vagina than in the cervix [16]. Most LR-HPV types in the present study belonged to Alpha-3 (HPV61, 81, 84) and Alpha-15 (HPV71, 90). These findings may support the conclusion that the vagina generally functions as a reservoir for many mucosal HPV types, 
and only HR- or pHR-HPV types can induce cancer if persistent infections with these types are established.

The ages of VaSCC patients were greater than those of VaIN cases in the present study (the average age of VaSCC cases was 81.7 years, which was greater than that of VaIN cases), suggesting long latency is needed for malignant progression. It was noted that six different HR- and pHR-HPV types were identified in each of the VaSCC cases, suggesting that various HPV types are responsible for VaSCC development. In particular, two of the six $(33.3 \%)$ cancers were infected with pHR types (HPV53, 67), suggesting that the prevalence of pHR types appears to be greater in VaSCC than in CxSCC [18]. Sakamoto et al. determined HPV type in CxSCC cases with the same method as this paper, the results revealed that he prevalence of HPV16 and 18 are lower in VaSCC than that in CxSCC $(p=0.019)$, although further study is needed to warrant it.

The present study also demonstrated that the manual microdissection procedure, which is a cost-effective method, is as effective as LCM for the acquisition of target tissue for HPV genotyping [20]. From a clinical perspective, the present study suggests that high-grade VaIN could be detected with a highly sensitive HPV test in cervical cancer screening and careful examination by colposcopy. Clinicians should also note that some VaIN lesions may be found in women who show false positive results in their cervix during screening. To reduce the incidence of cervical cancer in many countries, the nonavalent HPV vaccine, which protects against HPV6, 11, 16, 18, 31, 33, 45, 52, and 58 infections, has recently been launched [34]. The present study suggests that the nonavalent HPV vaccine could protect against $64.7 \%$ of VaIN2/3 and $42.9 \%$ of VaSCC in Japan. Similarly, the bivalent or quadrivalent HPV vaccines could protect against $41.2 \%$ of VaIN2/3 and $14.3 \%$ of VaSCC.

This study has some limitations. The most important of these is the small size of the samples of VaIN and, in particular, VaSCC, which comprised only seven cases. Thus, similar studies are needed to increase the sample size for further research.

\section{Conclusions}

Many HPV types, including high-risk (HR), possible high-risk (pHR), and low-risk (LR) types, were identified in low-grade vaginal intraepithelial lesion (VaIN1), whereas HR and pHR-HPV types were identified in high-grade vaginal lesions (VaIN2/3). The vagina appears to be the reservoir for any mucosal HPV type, and HR or pHR HPV types are causative agents for vaginal malignancies. Different HPV type distribution between the vagina and the cervix suggests that CIN and VaIN are independently developed in most cases.

Author Contributions: Conceptualization, T.S. and S.Z.; methodology, S.Z., M.S., K.O. and M.O.; validation, S.Z.; statistical analysis, S.Z.; pathological analysis, N.K., T.S. and J.S.; resources, T.S. and J.S.; data curation, T.S. and S.Z.; writing-original draft preparation, S.Z.; writing-review and editing, T.S. and S.Z.; visualization, S.Z.; supervision, T.S.; project administration, T.S.; funding acquisition, T.S. All authors have read and agreed to the published version of the manuscript.

Funding: This research received no external funding.

Institutional Review Board Statement: The study was conducted according to the guidelines of the Declaration of Helsinki, and approved by the Ethics Committee of Kanazawa Medical University (protocol code No.1408 and date of approval; 10 April 2019).

Informed Consent Statement: Informed consent was obtained from all subjects involved in the study.

Data Availability Statement: This study did not report any data.

Conflicts of Interest: The authors declare no conflict of interest. 




\section{References}

1. So, K.A.; Hong, J.-H.; Hwang, J.H.; Song, S.-H.; Lee, J.-K.; Lee, N.W.; Lee, K.W. The utility of the human papillomavirus DNA load for the diagnosis and prediction of persistent vaginal intraepithelial neoplasia. J. Gynecol. Oncol. 2009, 20, 232-237. [CrossRef] [PubMed]

2. zur Hausen, H. Papillomaviruses in the causation of human cancers-A brief historical account. Virology 2009, 384, 260-265. [CrossRef] [PubMed]

3. Segondy, M. Classification of papillomaviruses (HPV). Rev. Fr. des Lab. 2008, 38, 23-25. [CrossRef]

4. Van Doorslaer, K.; Li, Z.; Xirasagar, S.; Maes, P.; Kaminsky, D.; Liou, D.; Sun, Q.; Kaur, R.; Huyen, Y.; McBride, A.A. The Papillomavirus Episteme: A major update to the papillomavirus sequence database. Nucleic Acids Res. 2017, 45, D499-D506. [CrossRef]

5. Moscicki, A.-B.; Shiboski, S.; Hills, N.K.; Powell, K.J.; Jay, N.; Hanson, E.N.; Miller, S.; Canjura-Clayton, L.K.; Farhat, S.; Broering, J.M.; et al. Regression of low-grade squamous intra-epithelial lesions in young women. Lancet 2004, 364, 1678-1683. [CrossRef]

6. de Sanjosé, S.; Brotons, M.; Pavon, M.A. The natural history of human papillomavirus infection. Best Pr. Res. Clin. Obs. Gynaecol. 2018, 47, 2-13. [CrossRef]

7. Schiffman, M.; Castle, P.E.; Jeronimo, J.; Rodriguez, A.C.; Wacholder, S. Human papillomavirus and cervical cancer Burden of cervical cancer. Lancet 2007, 370, 890-907. [CrossRef]

8. Arbyn, M.; Tommasino, M.; Depuydt, C.; Dillner, J. Are 20 human papillomavirus types causing cervical cancer? J. Pathol. 2014 234, 431-435. [CrossRef]

9. Halec, G.; Alemany, L.; Lloveras, B.; Schmitt, M.; Alejo, M.; Bosch, F.X.; Tous, S.; Klaustermeier, J.E.; Guimera, N.; Grabe, N.; et al. Pathogenic role of the eight probably/possibly carcinogenic HPV types 26, 53, 66, 67, 68, 70, 73 and 82 in cervical cancer. J. Pathol. 2014, 234, 441-451. [CrossRef]

10. Halec, G.; Schmitt, M.; Dondog, B.; Sharkhuu, E.; Wentzensen, N.; Gheit, T.; Tommasino, M.; Kommoss, F.; Bosch, F.X.; Franceschi, S.; et al. Biological activity of probable/possible high-risk human papillomavirus types in cervical cancer. Int. J. Cancer 2013, 132, 63-71. [CrossRef]

11. Ganti, K.; Broniarczyk, J.; Manoubi, W.; Massimi, P.; Mittal, S.; Pim, D.; Szalmas, A.; Thatte, J.; Thomas, M.; Tomaić, V.; et al. The human papillomavirus E6 PDZ binding motif: From life cycle to malignancy. Viruses 2015, 7, 3530-3551. [CrossRef]

12. Thatte, J.; Massimi, P.; Thomas, M.; Boon, S.S.; Banks, L. The Human Papillomavirus E6 PDZ Binding Motif Links DNA Damage Response Signaling to E6 Inhibition of p53 Transcriptional Activity. J. Virol. 2018. [CrossRef]

13. Insinga, R.P.; Liaw, K.-L.; Johnson, L.G.; Madeleine, M.M. A systematic review of the prevalence and attribution of human papillomavirus types among cervical, vaginal, and vulvar precancers and cancers in the United States. Cancer Epidemiol. Biomark. Prev. 2008. [CrossRef]

14. Srodon, M.; Stoler, M.H.; Baber, G.B.; Kurman, R.J. The distribution of low and high-risk HPV types in vulvar and vaginal intraepithelial neoplasia (VIN and VaIN). Am. J. Surg. Pathol. 2006. [CrossRef] 
15. De Vuyst, H.; Clifford, G.M.; Nascimento, M.C.; Madeleine, M.M.; Franceschi, S. Prevalence and type distribution of human papillomavirus in carcinoma and intraepithelial neoplasia of the vulva, vagina and anus: A meta-analysis. Int. J. Cancer 2009, 124, 1626-1636. [CrossRef]

16. Zhang, S.; Saito, M.; Yamada, S.; Sakamoto, J.; Takakura, M.; Takagi, H.; Sasagawa, T. The prevalence of VAIN, CIN, and related HPV genotypes in Japanese women with abnormal cytology. J. Med. Virol. 2020. [CrossRef]

17. Mirkovic, J.; Howitt, B.E.; Roncarati, P.; Demoulin, S.; Suarez-Carmona, M.; Hubert, P.; McKeon, F.D.; Xian, W.; Li, A.; Delvenne, P.; et al. Carcinogenic HPV infection in the cervical squamo-columnar junction. J. Pathol. 2015. [CrossRef]

18. Sakamoto, J.; Kamiura, S.; Okayama, K.; Okodo, M.; Shibata, T.; Osaka, Y.; Fujita, S.; Takata, E.; Takagi, H.; Takakura, M.; et al. Single type infection of human papillomavirus as a cause for high-grade cervical intraepithelial neoplasia and invasive cancer in Japan. Papillomavirus Res. 2018, 6, 46-51. [CrossRef]

19. Van Der Marel, J.; Quint, W.G.; Schiffman, M.; Van De Sandt, M.M.; Zuna, R.E.; Dunn, S.T.; Smith, K.; Mathews, C.; Gold, M.A.; Walker, J.; et al. Molecular mapping of high-grade cervical intraepithelial neoplasia shows etiological dominance of HPV16. Int. J. Cancer 2012, 131, 946-954. [CrossRef]

20. Snow, A.N.; Stence, A.A.; Pruessner, J.A.; Bossler, A.D.; Ma, D. A simple and cost-effective method of DNA extraction from small formalin-fixed paraffin-embedded tissue for molecular oncologic testing. BMC Clin. Pathol. 2014, 14, 1-10. [CrossRef]

21. Okodo, M.; Okayama, K.; Teruya, K.; Sasagawa, T. Uniplex E6/E7 PCR method detecting E6 or E7 genes in 39 human papillomavirus types. J. Med. Virol. 2018, 90, 981-988. [CrossRef]

22. Saslow, D.; Solomon, D.; Lawson, H.W.; Killackey, M.; Kulasingam, S.L.; Cain, J.; Garcia, F.A.R.; Moriarty, A.T.; Waxman, A.G.; Wilbur, D.C.; et al. American Cancer Society, American Society for Colposcopy and Cervical Pathology, and American Society for Clinical Pathology screening guidelines for the prevention and early detection of cervical cancer. Am. J. Clin. Pathol. 2012. [CrossRef]

23. Mazellier, S.; Dadone-Montaudie, B.; Chevallier, A.; Loubatier, C.; Vitale, S.; Cardot-Leccia, N.; Angeli, K.; Trastour, C.; Delotte, J.; Giordanengo, V.; et al. Papillomavirus genotyping on formaldehyde fixed paraffin-embedded tissues in vulvar intraepithelial neoplasia. Arch. Gynecol. Obs. 2017, 296, 811-817. [CrossRef]

24. Sasagawa, T.; Maehama, T.; Ideta, K.; Irie, T. Population-based study for human papillomavirus (HPV) infection in young women in Japan: A multicenter study by the Japanese human papillomavirus disease education research survey group (J-HERS). J. Med. Virol. 2016, 88, 324-335. [CrossRef]

25. Liu, Y.; Alqatari, M.; Sultan, K.; Ye, F.; Gao, D.; Sigel, K.; Zhang, D.; Kalir, T. Using p16 immunohistochemistry to classify morphologic cervical intraepithelial neoplasia 2: Correlation of ambiguous staining patterns with HPV subtypes and clinical outcome. Hum. Pathol. 2017, 66, 144-151. [CrossRef]

26. Zhong, P.; Li, J.; Gu, Y.; Liu, Y.; Wang, A.; Sun, Y.; Lu, L. P16 and Ki-67 expression improves the diagnostic accuracy of cervical lesions but not predict persistent high risk human papillomavirus infection with CIN1. Int. J. Clin. Exp. Pathol. 2015, 8, $2979-2986$.

27. Calil, L.N.; Edelweiss, M.I.A.; Meurer, L.; Igansi, C.N.; Bozzetti, M.C. P16INK4a and Ki67 expression in normal, dysplastic and neoplastic uterine cervical epithelium and human papillomavirus (HPV) infection. Pathol. Res. Pr. 2014. [CrossRef]

28. Bertoli, H.; Rasmussen, C.L.; Sand, F.L.; Albieri, V.; Norrild, B.; Verdoodt, F.; Kjaer, S.K. Human papillomavirus and p16 in squamous cell carcinoma and intraepithelial neoplasia of the vagina. Int. J. Cancer 2019, 145, 78-86. [CrossRef]

29. Frega, A.; French, D.; Piazze, J.; Cerekja, A.; Vetrano, G.; Moscarini, M. Prediction of persistent vaginal intraepithelial neoplasia in previously hysterectomized women by high-risk HPV DNA detection. Cancer Lett. 2007. [CrossRef]

30. Tsimplaki, E.; Argyri, E.; Michala, L.; Kouvousi, M.; Apostolaki, A.; Magiakos, G.; Papassideri, I.; Panotopoulou, E. Human papillomavirus genotyping and E6/E7 mRNA expression in Greek women with intraepithelial neoplasia and squamous cell carcinoma of the vagina and vulva. J. Oncol. 2011, 2012. [CrossRef]

31. Chao, A.; Chen, T.-C.; Hsueh, C.; Huang, C.-C.; Yang, J.-E.; Hsueh, S.; Huang, H.-J.; Lin, C.-T.; Tang, Y.-H.; Liou, J.-D.; et al. Human papillomavirus in vaginal intraepithelial neoplasia. Int. J. Cancer 2012, 131. [CrossRef] [PubMed]

32. Quint, W.; Jenkins, D.; Molijn, A.; Struijk, L.; Van De Sandt, M.; Doorbar, J.; Mols, J.; Van Hoof, C.; Hardt, K.; Struyf, F.; et al. One virus, one lesion-Individual components of CIN lesions contain a specific HPV type. J. Pathol. 2012, 227, 62-71. [CrossRef] [PubMed]

33. Guimerà, N.; Lloveras, B.; Lindeman, J.; Alemany, L.; Van De Sandt, M.; Alejo, M.; Hernandez-Suarez, G.; Bravo, I.G.; Molijn, A.; Jenkins, D.; et al. The Occasional Role of Low-risk Human Papillomaviruses 6, 11, 42, 44, and 70 in Anogenital Carcinoma Defined by Laser Capture Microdissection/PCR Methodology. Am. J. Surg. Pathol. 2013, 37, 1299-1310. [CrossRef] [PubMed]

34. Joura, E.A.; Giuliano, A.R.; Iversen, O.-E.; Bouchard, C.; Mao, C.; Mehlsen, J.; Moreira, E.D.; Ngan, Y.; Petersen, L.K.; LazcanoPonce, E.; et al. A 9-valent HPV vaccine against infection and intraepithelial neoplasia in women. N. Engl. J. Med. 2014. [CrossRef] 